\title{
Driving device for a hand movement without external force
}

\author{
Esteban Peña-Pitarch, Neus Ticó Falguera, Joan Antoni Lopez Martinez, \\ Anas Al Omar, Iñaki Alcelay Larrión ${ }^{\mathrm{a}}$ \\ ${ }^{a}$ Escola Politècnica Superior d'Enginyeria de Manresa (UPC) \\ Av. Bases de Manresa, 61-73 \\ (08240) Manresa, Spain
}

\begin{abstract}
People with disabilities have limitations in activities of daily life such as grasping a glass of water or moving an object. Orthotic products that improve or restore the functionality of the musculoskeletal system of a patient contribute to some extent to overcome the limitations described. So does the hand brace, used to treat musculoskeletal disorders caused by various diseases (rheumatic disorders, neurological, orthopedic and others). The paper simulates a novel exoskeleton helping to grasp any object. The novelty of this mechanism is that works without external energy, it works with a wrist movement that generates a kinetic movement and helps to grasp objects with an extra force. The orthosis facilitates the functionality, being comfortable and easy to be used by the patient. It is adaptable to hand size and finger length of the patient.
\end{abstract}

Keywords: exoskeleton, force, grasp, orthosis, unpowered

\section{Introduction}

Hand partial disabilities become a serious problem for many people, who neither have mobility and feeling in their hands, nor enough strength to support objects such as a simple glass of water. Mainly elderly people suffer this problem, but also many people who have suffered accidents and people with certain types of musculoskeletal conditions such as osteoarthritis.

However, the technology developed has the novelty of being comfortable and easy to be used by the patient. It is adaptable to hand size and finger length of the patient and needs no power source for operation. 
For several decades the development of a new hand brace has included new technological advances. However, there is still a significant gap between the state of the art equipment designed in terms of presenting a combination of highly functional, durable, aesthetically and economically viable [5]. In this regard, a number of factors are critical in the design of actuators for a hand.

In principle the design of the device used will be taken into account and therefore the device will be adapted to the size of the patient's hand and will have a suitable weight so that it can be used comfortably.

Furthermore the design must consider what is the best operating mechanism of the device in terms of type of actuator (manual or electric), the gripping force provided by the apparatus and the gripping speed thereof. Finally, the design will have to evaluate which method is the union of the joints and the type of grip that provides special emphasis on the thumb because it is responsible for $40 \%$ of full functionality of ones hand [16]. In this sense, the technology developed in this paper takes into account the following: It can be adapted to different hand sizes of patients and amplifies grip strength in addition to not requiring any external power source (no batteries or batteries, or Electromyography (EMG)) and is driven only by the flick of the wrist. Therefore, the technology developed can be easily and conveniently used by patients when performing daily activities. It is easy to manufacture and it is economically competitive since it does not require complex mechanical components, which can make the technology developed one of the key factors when it comes to be funded by the National Health Service (NHS).

There are a variety of devices for a hand operation that enable or facilitate the movement or function of it. Orthosis is an orthopedic appliance or apparatus used to support, align, prevent, or correct deformities or to improve the function of movable parts of the body. Based on this definition, our research is focused on the development, design, and building of an exoskeleton, so named our orthosis. This device needs to follow four initial premises: a) all the components could be built by the users, b) could be used without any external forces, c) aesthetic appearance and ergonomic aspect, and d) the target people to used it are those with little hand mobility and without force, such as elderly or people who suffer from some pathologies.

Under these premises we design and build an exoskeleton that works with a wrist movement and helps to grasp any object used in the active daily live (ADL). We consider two types of grasp: power grasp, when grasp using the palm, and precision grasp when grasp using the fingertips, defined in [18]. 
One model of an orthotic hand that has been investigated consists on an exoskeleton with four degrees of freedom for the rehabilitation of the index finger. The device can generate bidirectional motion (flexion-extension) for all joints of the finger and it is adjustable for various hand sizes. Force sensors such as FlexiForce and encoders with a DC motor are used to measure the angular position. The information received from the sensors is used to control the exoskeleton and to evaluate and analyze the effects of rehabilitation [19]. One more anthropomorphic hand was presented. The problem with this design is that comprises a DC motor since it uses a power source; therefore, the exoskeleton does not have a prolonged time of use [1].

Another orthotic model studied was an exoskeleton for hand rehabilitation [20], a mechanical prototype with four degrees of freedom, which is moved by an actuating unit and receives information through the Hall effect sensors in each joint structure. This model can also use resistive force sensors at the top and bottom of the phalanges and myoelectric sensors to measure the activity of some muscles of interest. The problem with this type of hand brace lies not only in the need for an external power source but can also pose a greater weight to the patient's hand where it is applied.

An orthotic hand for preventive purposes was also designed [14]. In this sense, it highlights a prototype exoskeleton designed to fit into the gloved hand of an astronaut and to counteract the rigidity of the pressurized space suit. The hand movements are monitored with an array of pressure sensors located between the exoskeleton and the hand. By means of a microcontroller, the commands apply to a driver under Pulse With Modulation (PWM) controlled motor.

Finally, other prototypes were designed in order to create virtual environments with which to interact. In this area, some authors have proposed a new methodology for master-slave systems, using passive force feedback [5] to build an exoskeleton master hand with three fingers and four degrees of freedom (DOF) each, using force feedback and a control algorithm that uses electromagnetic clutches and elastic elements. Its operation is based on a switch between force control and position control, and this switching depends directly on whether it is in contact with an object. With the exoskeleton built and implemented control strategy we can design a virtual reality system for the hand. In the same direction, an exoskeleton proposed by other authors allows full extension and flexion of the fingers and thumb [16]. Some of them apply a two-way feedback, and others, three DOF for the index and four DOF for the thumb. They use direct current motors, power 
cables and force sensors to measure the power of the actuators and the ability to force one's hand. The facility is designed to be used in conjunction with a commercial haptic arm with six DOF, in order to enable simulation of external forces.

A technique to stimulate finger pad shear deformation transferred to the side of the fingertip was developed by [6]. However, [8] developed a wearable sensor system for estimating finger contact force by measuring the mechanical deformation of the side of the finger pad. A new model to simulate contact and rolling motion between two soft fingers and an object by using Finite Element Method (FEM) and constraint stabilization methods. In similar line [9] presents a hemisphere-shaped soft finger for soft fingers, based on force distribution.

Pressure distribution on the hand surface during hand grasping an elliptic cylindrical handle was study by [17] based on computer aided engineering analysis. [21] presented a study intended to analyze, theoretically, the time dependent deformation profile of skin surface, the strain distributions within soft tissue, and the response force of a fingertip when it is stimulated by a probe vibrating with a sinusoidal movement. In order to simulate the skin contact deformation mechanism, a physical model based on the Boussinesq approximation with nonlinear elasticity and a compressing-swelling effect was proposed by [22].

[13] showed a complete grasp model used to simulate an experiment in which a subject was asked to grasp two cylinders of different diameters and weights. It showed similar angles in the experiments to the ones that we calculate with our approximation. Similar results are also shown when using objects with 3,5 , or $7 \mathrm{~cm}$ in diameter in [3]. Using an instrumented cylinder [4] described the magnitude of pinch force as the function of a cylinder allowing simultaneous measurements of the opposition axis of the index and the thumb of the hand. [2] have introduced a paradigm in which subjects grasp from the same starting position to the same final object, once as a typical laboratory task and once as a part of everyday-like behavior.

This paper is organized as follows: Section 2 shows the device. Section 3 introduces results and discussion. Finally conclusions are given in section 4 .

\section{Device}

According to the survey Disability, Personal Autonomy and Dependency of National Institute of Statistical (INE 2008, Spain), people with disabili- 
ties have limitations associated with doing housework, chores and personal hygiene care and with moving, grasping, and transporting objects. There are a variety of devices to drive either a prosthesis or an orthosis that enable or facilitate the functionality of the same devices as the type of continuous passive motion (CPM), which are preferably used for rehabilitative purposes.

The technology developed in this paper is part of an innovative project in the field of the design of an exoskeleton to facilitate extra force of the fingers of one hand to people who are not strong enough to support objects, such as the elderly, people who have suffered accidents or people with certain diseases.

Figure 1 shows a first design of the exoskeleton. The next design simplify the exoskeleton to build and use more usefully by the users.

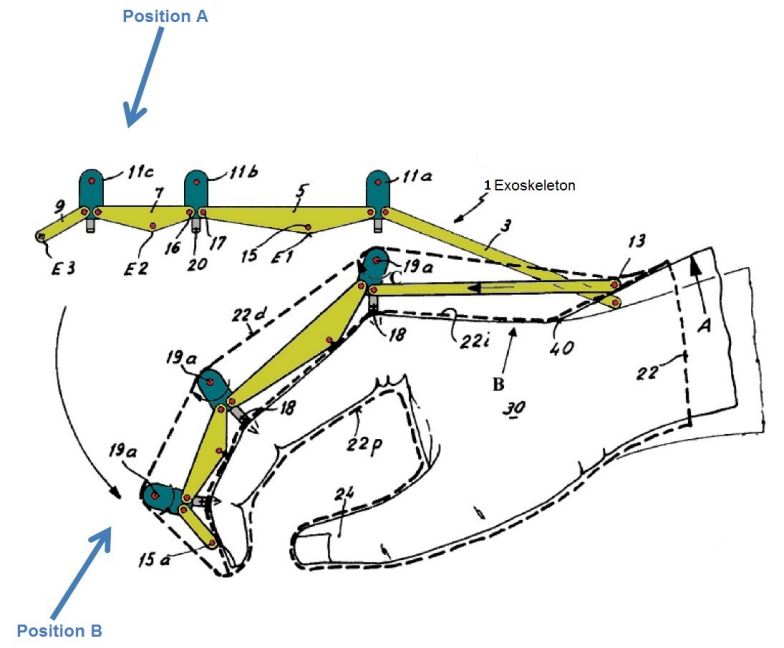

Figure 1: First exoskeleton design to adjust in neutral hand position.

We can observe in Figure 2 a three dimension (3D) draw of the exoskeleton designed. Segments $(3,5)$ are designed and built for each finger, index, middle, ring, and small. The length of each finger depends on the hand length (HL), the relationship between the length of each phalanx and the HL can be found in [10]. For each finger, exoskeleton has a first segment (3) and a second one (5) corresponding to the bones proximal phalanx (3), medial phalanx, and distal phalanx (5). Thanks to an intermediate part the exoskeleton consists of several hinge plates (11). Each element (11) is 
fastened to its correspondenting finger and there are four for finger. Element (12) connect the segments $(3,5)$ and are jointed by a revolute joint in two parts, the upper part is attached to the fixed element (11) and permit tilt the angle necessary to balance the segment attached, in the lower part the segment (3) or (5) are attached.

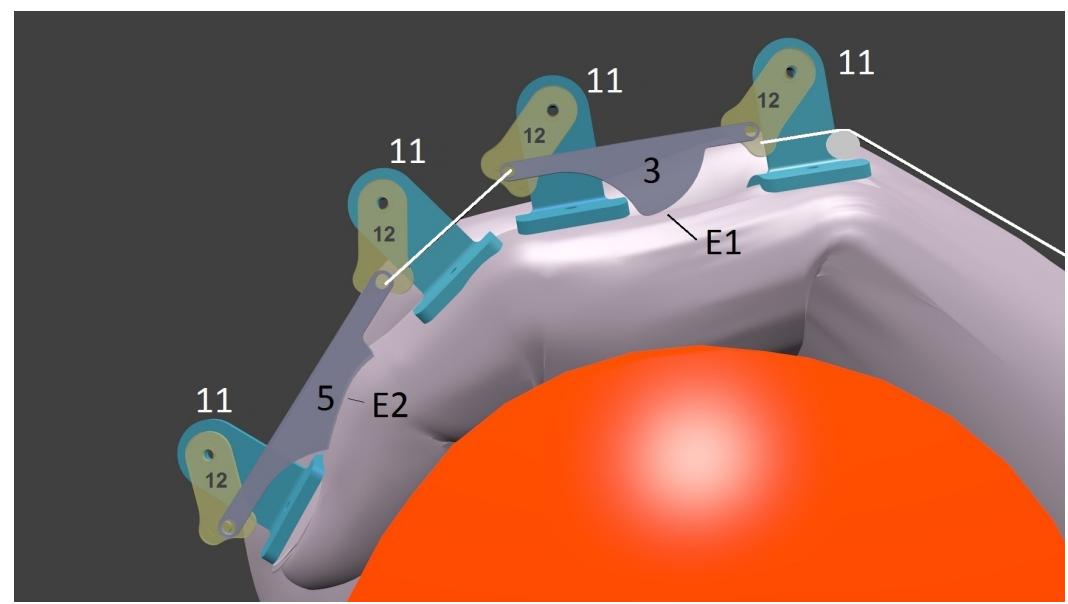

Figure 2: Exoskeleton, position in neutral hand position.

Considering the aforementioned exoskeleton applied to the dorsal hand, the segments are parallel to these bones and phalanges and consist essentially of elongated rigid strips, being first (3) and second (5) segments directed towards the dorsal surface of the corresponding finger. These segments have protruding portions of thrust (E1, E2), shown in Figure 2, and together with another portion of thrust are in contact with the corresponding finger to push it in the middle of the phalanx not matching the knuckles when the mobile exoskeleton moves, triggering the simultaneous movement of the segments (3 and 5). Rotation is transmitted through the intermediate (joint plates 11) around the respective joint (19) so that the plate is directed to a second position.

The hinged support plates (11) as shown in Figure 3A-shaped pillar, comprise each of the joints (19) configuration at the free end of the support (11) in the form of pins and some set in the form of additional holes (19) arranged next to one end of each of the joint plates (11). In the example illustrated in Figure 3B each of these plates, elongated like a tab in the palmar-dorsal direction of the hand, forms a hollow casing with its respective holes (19), 
A

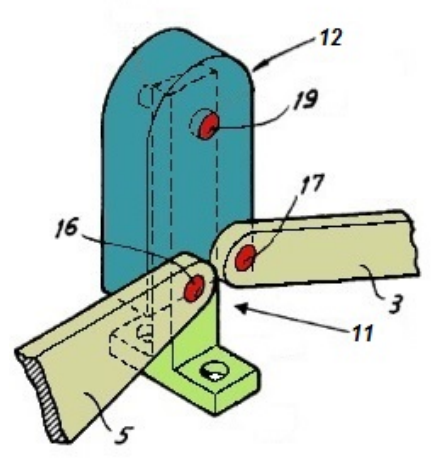

B

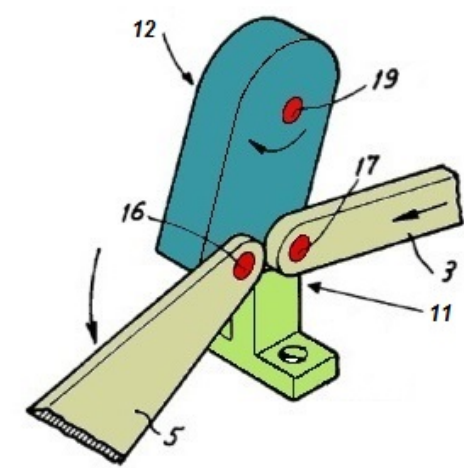

Figure 3: Initial position (B) and activated position (A).

one on each side higher although other than the illustrated example. The plates are solid and only have a larger face with a through hole (19) to be inserted in a corresponding pin (19) of the support (11). Two pivots (16, 17) are articulated in their corresponding holes at the end of two consecutive segments, as illustrated in Figure 3, in relation to the first (3) and second (5) segments.

To activate the exoskeleton, once it is fitted in the hand by the user, the movement of extension of the wrist generates a movement of the plate (11) and this movement is transmitted to the others plates for each finger, through cables or rigid bars. To deactivate, the exoskeleton returns to the neutral position with a movement of flexion of the wrist.

Figure 4 shows the mechanism used to activate the movements of the plates (12). The trigger is in contact with the metacarpal bones of the dorsal surface of the hand. Before grasping, the mechanism follows the movement of the hand and fingers. Once the person is feeling the object to grasp, one movement of the wrist flexion permits to have an extra force. When the wrist rotates the rotational spring go to the neutral position and the device is deactivated.

The mechanism is built as a four-bar linkage, with two four-bar linkage for finger as shown in Figure 2. Plates (12) have the same length and the movement of the elements ( 3 and 5 ), have a curvilinear translational movement. For this reason, the kinematics of the mechanism are avoided. Plates 
(12) are working in compression and the maximum force is $1.5 \mathrm{~N}$. The spring function is restoring the neutral position of exoskeleton with an insignificant resistance. The idea is to built a commercial spring with small dimensions, just for restore to neutral position.


Figure 4: Mechanism used to activate/desactivate exoskeleton.

The mechanism is covered by an aesthetic glove and it is easy to be used.

\section{Results and Discussion}

This patent born from the research in virtual human hand and rehabilitation under stroke. For this reason before proceeding with the design, we have dates and have validated these dates. In this section, we show how the data was obtained and how we can apply to the exoskeleton to demonstrate that it work.

In the Section 2, we show how the device work, but for the design shown the question is: is the movement of the wrist it is enough to give the force to the user to grasp any object in the activity of daily life (ADL)?. The answer is yes, and for that we demonstrate that the force exerted for each point of contact (E1, E2) is sufficient to grasp. Next we focus in the finger deformation and based in these deformation we calculate the force exerted.

\subsection{Finger deformation}

When the exoskeleton is activated the extra-force is obtained by the movement of the finger. This force can be measured by the finger deformation and we calculated it with a FEM method.

The finite element method has been applied to the computer model developed. Figure 5 shows graphically the results of the strain obtained in both cases using the finite element method and the experiment carried out with 

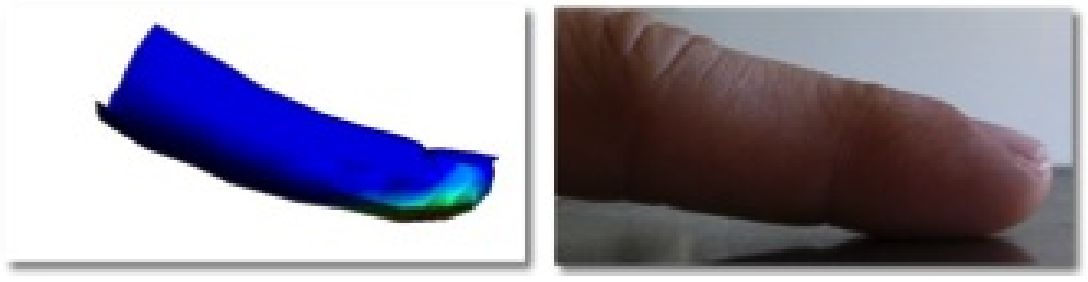

Figure 5: Finite element method simulation and experimental model.

an actual finger, when applying a vertical force directed towards the index finger of $1 \mathrm{~N}$.

Since each person has different finger settings, each finger has a different Poisson's ratio and a different Young's modulus. Therefore, these deformations have been calculated with different Poisson ratios, keeping constant Young's modulus and viceversa, i.e. by varying the Young's modulus and Poisson ratio maintaining constant, no large variations observed by giving the data obtained [15].

After calculating the deformation of the fingertip, Figure 6 shows the beam to a segment of the finger. The colored part shows the initial position of the exoskeleton, with a starting position of 45 degrees. When applied to the wrist motion, the apparatus begins to compress the finger up to 3 $\mathrm{mm}$. This deformation is applied to the fingertip. Since this deformation, according to the established model calculations, corresponds to a force of $5 \mathrm{~N}$, if we transfer this force to each segment of the finger, i.e., the distal, middle, and proximal, we can say that each finger is applied force of $15 \mathrm{~N}$, when grasping with power, shown in the Figure 7 and if all of them act, the fingers can exert a force of $60 \mathrm{~N}$. The thumb only gives our support mechanism without applying force.

The table 1 shows the comparison of the results obtained by the experiments [11], and the results of the finite element calculation.

The results obtained by the calculation show a pattern similar to the experimental data. The displacements produced may not be exactly equal for both methods, since the experimental method can have a margin of error having to force oneself to be difficult to maintain the same force for a few seconds while measuring displacement. Therefore, the 3 replicates were performed for each measurement. However, it is difficult to make accurate displacement. Therefore, small differences obtained between both methods 


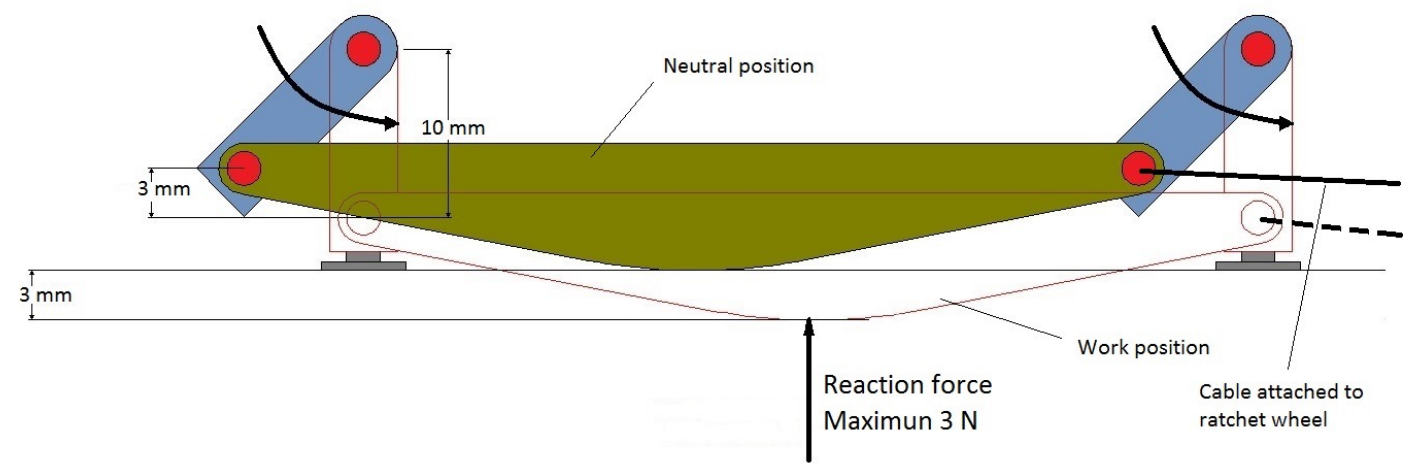

Figure 6: A maximum displacement of the segments of the finger.

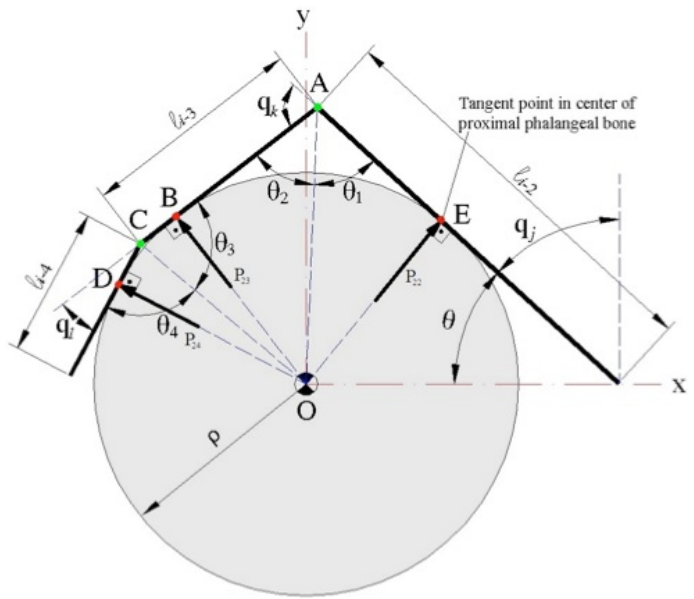

Figure 7: Grasping object with power grasp. 
Table 1: Fingertip deformation for each force in mm. Experimental and FEM.

\begin{tabular}{|c|c|c|}
\hline FORCE $(\mathrm{N})$ & EXPERIMENTAL $(\mathrm{mm})$ & FEM $(\mathrm{mm})$ \\
\hline 1 & 0.92 & 0.72001 \\
\hline 2 & 1.39 & 1.3258 \\
\hline 3 & 1.857 & 1.7644 \\
\hline 4 & 2.36 & 2.284 \\
\hline 5 & 2.867 & 2.8006 \\
\hline 6 & 3.33 & 3.2508 \\
\hline
\end{tabular}

can be considered the model valid.

Figure 8 shows an exoskeleton prototype. This prototype is built with a steel cut by laser machine. To see the exoskeleton prototype we remove the aesthetic globe.

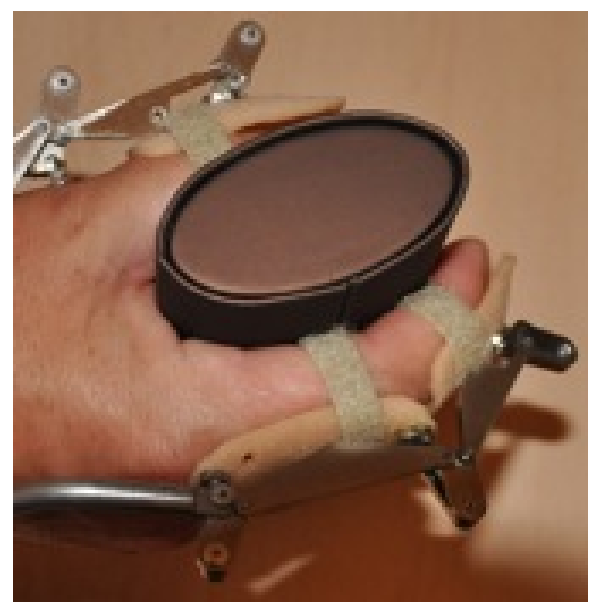

Figure 8: Exoskeleton prototype.

\subsection{Grasping with the device}

To follow the movement of the hand the device is attached and fixed to the hand. When the user grasps the object with a wrist movement, the exoskeleton is activated and give an extra force helping to grasp an object. Grasping is described in [12], but our interest now is to find out if the forces 
and movements when grasping the object with the help of the exoskeleton are sufficient to grasp the object with the security that it is possible to do the task, for instance, drinking a glass of water.

To validate if our device can grasp an object to do the activities of daily life, we need to calculate the forces and moments exerted by the deformation of the fingertip, with a maximum deformation of 3 millimeters.

\subsection{Grasp Statics}

Once the hand with the exoskeleton grasps an object we consider it an static problem, because we do not take into account of the manipulation of the object in this case. With this assumption, we apply the grasping equations shown in [7].

We use the grasp distribution for a sphere with precision shown in Figure 7 and we consider soft-finger. The grasp distribution for soft-finger from the equation:

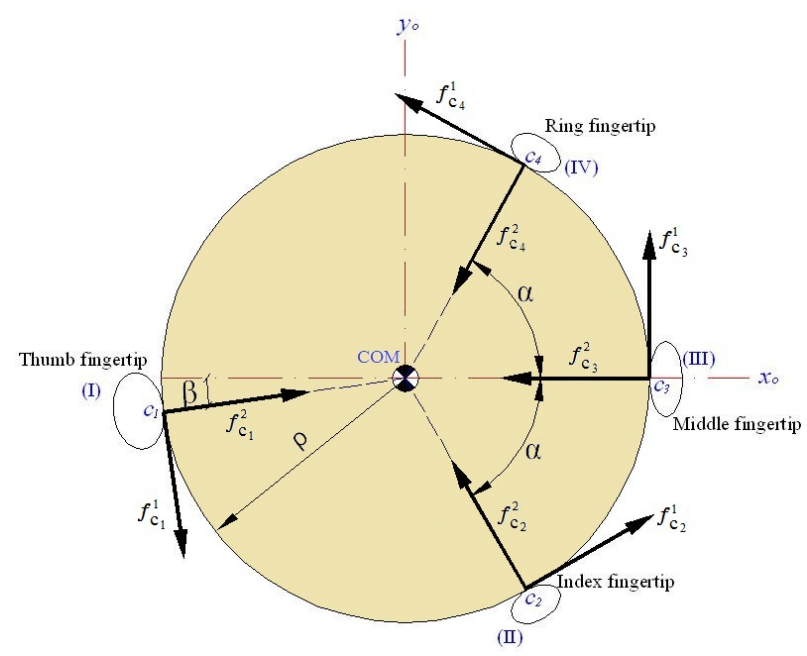

Figure 9: Grasping contact points and local axes

$$
G_{i}=\left[\begin{array}{cc}
R_{c_{i}} & 0 \\
\widehat{p}_{c_{i}} R_{c_{i}} & R_{c_{i}}
\end{array}\right]
$$

where $c_{i}$ is the contact point

$i$ subindex is for the finger contact, the maximum is $i=14$. 
$\widehat{p}_{c_{i}}$ is the skew-matrix for the position object c.o.m (center of mass)(COG) $\left(x_{o}, y_{o}, z_{o}\right)$ coordinates object center of mass and the grasp matrix is,

$$
G=\left[G_{1}\left|G_{2}\right| G_{3}\left|G_{4}\right| \ldots \mid G_{14}\right]
$$

with $G \in \Re^{6 x 56}$. For the contact forces

$$
f_{c_{i}}=\left\{f_{c_{i}}^{1}, f_{c_{i}}^{2}, f_{c_{i}}^{3}, f_{c_{i}}^{4}\right\} \in \Re^{56}
$$

where $i=1 \ldots 56$

where the super script ${ }^{2}$ indicates the normal forces, ${ }^{1}, 3$ indicate frictional forces, and ${ }^{4}$ is the torsional moment, and the friction cone for each contact finger,

$$
F C=F C_{c_{1}} \cdot F C_{c_{2}} \cdot F C_{c_{3}} \cdot F C_{c_{4}} \ldots F C_{c_{56}}
$$

where,

$$
F C_{c_{i}}=\left\{f_{c}: \sqrt{\left(f_{c_{i}}^{1}\right)^{2}+\left(f_{c_{i}}^{3}\right)^{2}} \leq \mu f_{c_{i}}^{2},\left|f_{c_{i}}^{4}\right| \leq \gamma f_{c_{i}}^{2}, f_{c_{i}}^{2} \geq 0\right\}
$$

where $\mu$ is the static coefficient of friction and $\gamma$ is the coefficient of torsional friction.

From the definition of [7] grasp is a force-closure if given any external wrench $\omega_{e x t} \in \Re^{p}$ applied to the object, there exist a contact forces $f_{c} \in F C$ such that

$$
G \cdot f_{c}=-\omega_{e x t}
$$

\subsection{Grasping forces}

Once the equations are applied next figures show the forces obtained and demonstrate that the object with a weight of $10 \mathrm{~N}$ can be grasped with the help of the exoskeleton presented in this paper.

Normal forces $f_{c_{1}}^{2}=f_{c_{2}}^{2}=f_{c_{3}}^{2}=f_{c_{4}}^{2}$ are showed in table 1 and depend of the fingertip deformation, for $3.25 \mathrm{~mm}$ the normal force is $6 \mathrm{~N}$, for $2.28 \mathrm{~mm}$ the normal force is $4 \mathrm{~N}$, and so on.

The next two figures 10, 11 show the friction forces calculated with the equations obtained, for the contact $c_{2}$. The isolines represent the friction forces, horizontal coordinate is the weight of the object and vertical coordinate is the normal force, the intersection of both lines is the friction force for the contact point $c_{i}$. To graph these forces, first we calculate the normal forces with the finite element method and the weight of the object is known and change from 4 to $10 \mathrm{~N}$. 


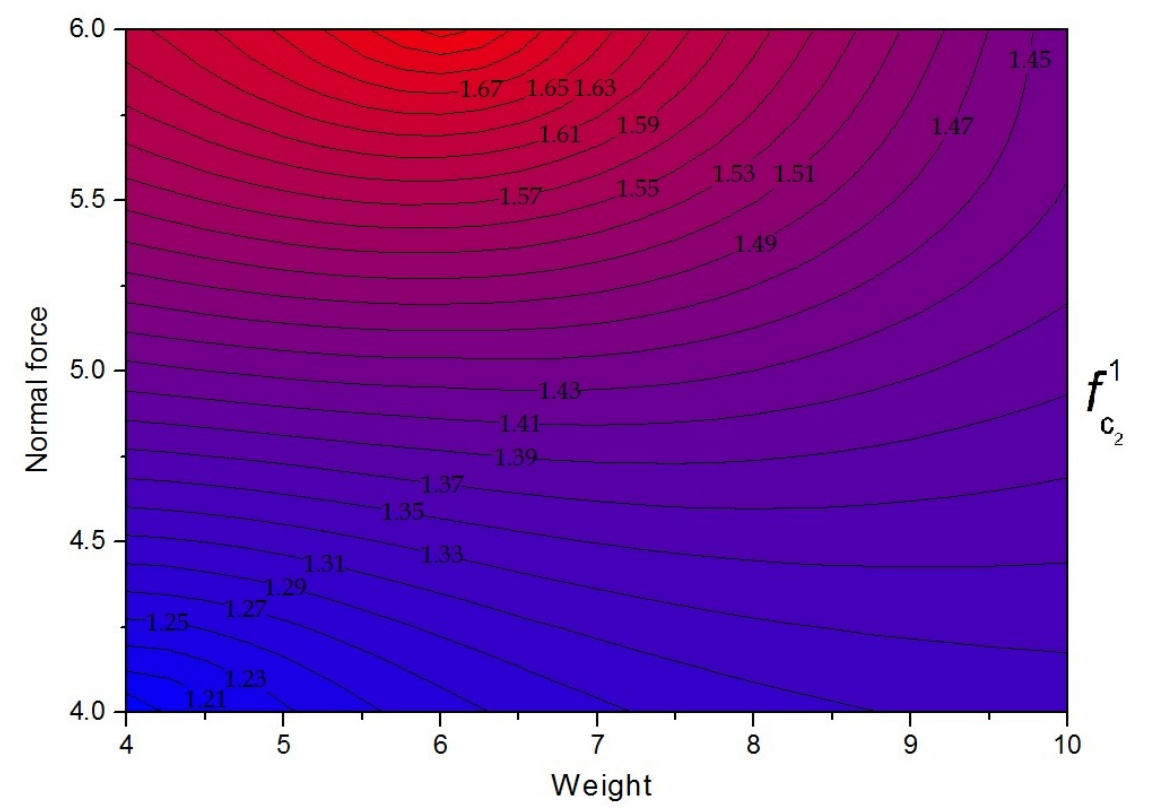

Figure 10: Friction force calculated for the contact point $c_{2}$. Index finger

\section{Conclusions}

Helping people with some pathology or elderly to grasp any object is possible with the exoskeleton designed in this paper. Only with the movement of the wrist the exoskeleton can generate a movement and push in a contact point to generate an extra force when grasping any object. The novelty of the exoskeleton is that it doesn't need any power source to work, only the movement of the wrist.

It is designed to build with a 3D printer and adjusted to any hand length. It is comfortable and easy to be used. We calculate the joint angles and these angles are compatible with the movement of the hand.

The extra force exerted when the exoskeleton is working is sufficient to grasp any object used in the ADL.

The simple design and building permit its use in any of the hands, left or right. 


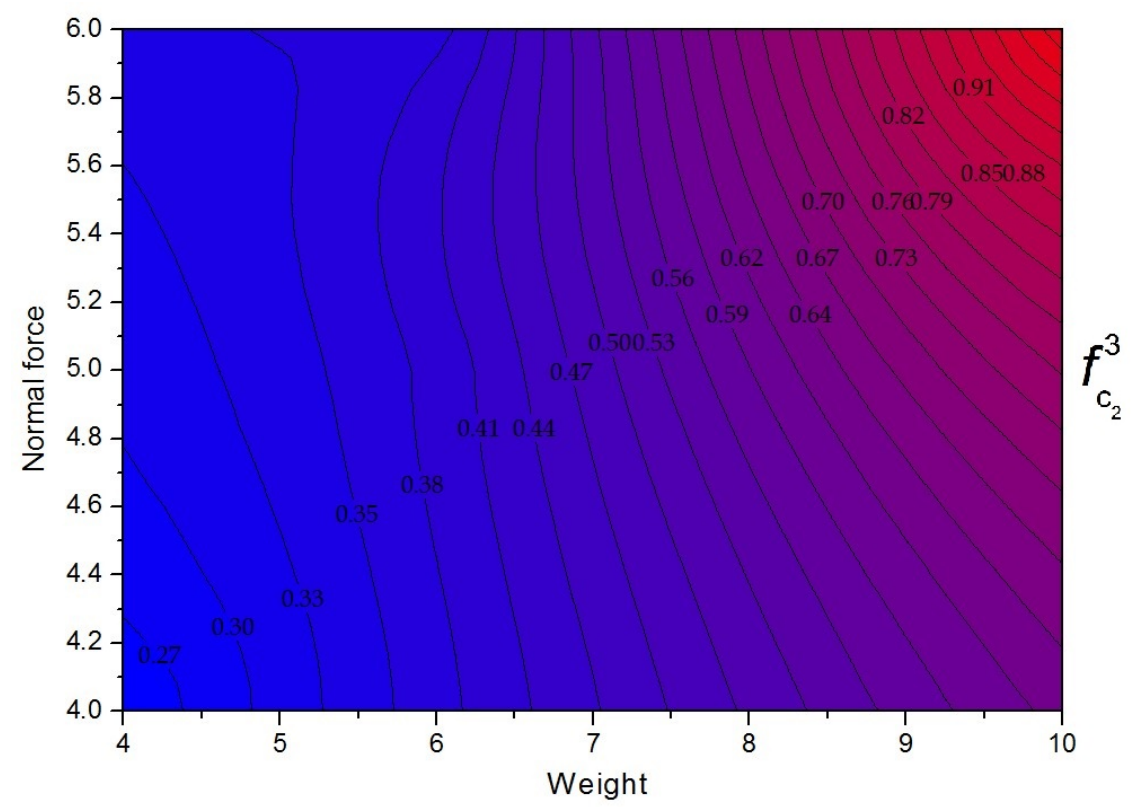

Figure 11: Friction force calculated for the contact point $c_{2}$. Index finger

\section{Acknowledgement}

This work was partially supported by the Spanish government with the project DPI2013-40882-P.

\section{References}

[1] Belter, J. T., Dolllar, A. M., Switzerland 2011. Performance characteristics of anthropomorphic prosthetic hands. IEEE International Conference on Rehabilitation Robotics.

[2] Bock, O., Züll, A., Feb. 2013. Characteristics of grasping movements in a laboratory and in an everyday-like context. Human movement science 32 (1), 249-56.

[3] Bongers, R. M., Zaal, F. T. J. M., Jeannerod, M., Jun. 2012. Hand aperture patterns in prehension. Human movement science 31 (3), 487501. 
[4] Bourbonnais, D., Frak, V., Pilon, J.-F., Goyette, M., Jan. 2008. An instrumented cylinder measuring pinch force and orientation. Journal of neuroengineering and rehabilitation 5, 2 .

[5] Koyoma, T., Yamano, I., Takemura, K., Maeno, T., 2002. Multi-fingered exoskeleton haptic device using passive force feedback for dexterous teleoperation. In: Intl. Conference of Intelligent Robots and Systems, Switzerland.

[6] Matsuura, Y., Okamoto, S., Yamada, Y., October 2014. Estimation of Finger Pad Deformation based on Skin Deformation Transferred to the Radial Side. Lecture Notes in Computer Science 8619 (25135717), 313319.

[7] Murray, R. M., Li, Z., Sastry, S., 1994. A Mathematical Introduction to Robotic Manipulation. No. ISBN 0-8493-7981-4. CRC Press.

[8] Nakatani, M., Kawasoe, T., Shiojima, K., Koketsu, K., Kinoshita, S., Wada, J., 2011. Wearable contact force sensor system based on fingerpad deformation. 2011 IEEE World Haptics Conference, WHC 2011, 323328 .

[9] Park, K.-H. P. K.-H., Kim, B.-H. K. B.-H., Hirai, S., 2003. Development of a soft-fingertip and its modeling based on force distribution. 2003 IEEE International Conference on Robotics and Automation (Cat. No.03CH37422) 3, 3169-3174.

[10] Peña Pitarch, E., 2008. Virtual human hand: Grasping strategy and simulation. Ph.D. thesis, Universitat Politecnica de Catalunya.

[11] Peña Pitarch, E., Ticó Falguera, N., Vinyes Casasayas, A., Martinez Carmona, D., July 2012. Fingertips deformation under static forces: Analysis and experiments. In: House, C. P. (Ed.), International Conference on Applied Human Factors and Ergonomics. Vol. 2012 AHFE Internacional Conference. pp. 4618-4626.

[12] Peña Pitarch, E., Ticó Falguera, N., Yang, J. J., 2014. Virtual human hand: model and kinematics. Computer Methods in Biomechanics and Biomedical Engineering 17 (5), 568-579. 
[13] Sancho-Bru, J. L., Mora, M. C., León, B. E., Pérez-González, A., Iserte, J. L., Morales, A., May 2012. Grasp modelling with a biomechanical model of the hand. Computer methods in biomechanics and biomedical engineering (April 2013), 37-41.

[14] Shields, B., Main, J., Peterson, S., Strauss, A., 1997. An anthropomorphic hand exoskeleton to prevent astronaut hand fatigue during extravehicular activities. IEEE Transactions on Systems, Man and Cybernetics,Part A: Systems And Humans 27 (5), 668-673.

[15] Shimawaki, S., Sakai, N., 2007. Quasi-static Deformation Analysis of a Human Finger using a Three-dimensional Finite Element Model Constructed from CT Images. Journal of Environment and Engineering $2(1), 56-63$.

[16] Stergiopoulos, P., Moreau, G., Ammi, M., Fuchs, P., 2003. A framework for the haptic rendering of the human hand. In: In Proceedings of HAPTICS. pp. 340-347.

[17] Toet, a., Van Erp, J. B. F., Petrignani, F. F., Dufrasnes, M. H., Sadhashivan, a., Van Alphen, D., Boeree, F., De Gruijter, H. O., Hoeksema, J., Stamhuis, C. T., Steenbergen, P. J., 2013. Reach out and touch somebody's virtual hand: Affectively connected through mediated touch. Proceedings - 2013 Humaine Association Conference on Affective Computing and Intelligent Interaction, ACII 2013, 786-791.

[18] Tubiana, R., 1981. The hand. Volume I, 2nd Edition. W.B. Saunders Company.

[19] Wang, J., Li, J., Zhang, Y., Wang, S., 2009. Design of an exoskeleton for index finger rehabilitation. In: Proceedings of the 31st Annual International Conference of the IEEE-EMBS, Minneapolis, USA.

[20] Wege, A., Kondak, K., Hommel, G., 2005. Mechanical design and motion control of a hand exoskeleton for rehabilitation. In: Proceedings of the International IEEE Conference on Mechatronics and Automation (ICMA), Canada.

[21] Wu, J. Z., Dong, R. G., Schopper, a. W., Smutz, W. P., 2003. Analysis of skin deformation profiles during sinusoidal vibration of fingerpad. Annals of Biomedical Engineering 31, 867-878. 
[22] Xie, Y., Kanai, S., Date, H., 2013. Simulation of contact deformation property of digital hand skin and its experimental verifications. Key Engineering Materials 530, 675-680. 